\title{
Hilar Portion of the Left Hepatic Duct
}

National Cancer Institute

\section{Source}

National Cancer Institute. Hilar Portion of the Left Hepatic Duct. NCI Thesaurus. Code C38670.

The segment of the left hepatic duct located in the hilum of the liver. 